Beratungszahlen zu steigern. Zudem sind die in den Erhebungsbögen erfassten Daten von den Einrichtungen selbstberichtet und damit ist eine Unschärfe durch Überschätzung nicht auszuschließen. Von großer Bedeutung ist allerdings, dass die erfassten Daten nichts über die Qualität der Beratung aussagen - geringere Beratungsprozentsätze müssen nicht unweigerlich Folge einer unzureichenden oder schlechteren Beratung sein.

Die hier dargestellten Ergebnisse sollten also mit Vorsicht interpretiert werden. Das Fehlen von personenbezogenen Daten macht es nämlich unmöglich zu bestimmen, welche Patientencharakteristika mit einer Inanspruchnahme korrelieren. So kann z. B. aus den Erhebungszahlen nicht geschlossen werden, ob Patienten mit einem höheren Bedarf nach psychosozialer Beratung dementsprechend auch häufiger Unterstützung erhalten haben.

Es ist wichtig für die psychosoziale Versorgungsforschung, das Verhältnis zwischen

\section{„Geringere Beratungsprozentsätze müssen nicht unweigerlich Folge einer unzureichenden oder schlechteren Beratung sein."}

Angebot und Nachfrage mit in derartige Analysen einfließen zu lassen und zu prüfen, ob die Patienten wirklich den Wunsch nach einer professionellen Beratung verspüren und sich der verschiedenen Angebote in den Zentren wirklich bewusst sind.

Das Einbeziehen personenbezogener Daten wird helfen, sowohl die individuellen Bedarfe der Patienten als auch sozioökono- mische Merkmale und Indikatoren zu untersuchen, die eine mögliche Unterversorgung spezifischer Populationsgruppen zur Folge haben. Es gilt für die Zukunft, bestehende Barrieren aufzudecken und zu eliminieren - schließlich soll jedem Patienten, unabhängig vom Zentrum in dem er behandelt wird und unabhängig von persönlichen Merkmalen, eine qualitativ gleich gute psychosoziale Beratung niederschwellig und leicht zugänglich ermöglicht werden.

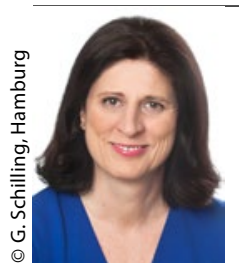

PD Dr. med. Georgia Schilling Hamburger Krebsgesellschaft e.V. g.schilling@ krebshamburg.de

\title{
Depressionen bei pflegenden Angehörigen nicht unterschätzen
}

\section{Psychisches Wohlergehen pflegender Angehöriger und die vom Patienten berichtete Pflegequalität sind eng verknüpft. US-amerika- nische Wissensschaftler überprüften diesen Zusammenhang am Beispiel von Depressionssymptomen bei Pflegenden.}

D ie vom Patienten wahrgenommene Pflegequalität wird durch seine Lebensqualität oder auch ein geringes Vertrauen in die Behandler beeinflusst. Inwieweit pflegende Angehörige oder Freunde, die auch bei onkologischen $\mathrm{Pa}$ tienten oftmals einen Großteil der Betreuung übernehmen, Einfluss auf die wahrgenommene Pflegequalität haben, wurde bisher noch wenig untersucht; dabei spielen sie oft eine wichtige Rolle, nicht nur in der psychischen Betreuung sondern auch bei der Koordination der medizinischen Betreuung. Auch ihre Stimmung und Befindlichkeit dürfte die vom Patienten wahrgenommene Pflegequalität mit bestimmen. Diese Fragestellung wurde jetzt im Rahmen einer Studie mit Daten des Cancer Care Outcomes Research and Surveillance (CanCORS) Konsortiums wissenschaftlich überprüft.

In die Untersuchung einbezogen wurden 689 Patienten mit einem neu diagnostizierten Lungen- oder Kolorektalkarzinom sowie jeweils eine von den $\mathrm{Pa}$ tienten benannte nahe stehende BezugsStörvariablen person. Anhand von multivariaten logistischen Regressionsanalysen wurden dann u.a. die Patientenangaben zur Qualität ihrer medizinischen Versorgung in Beziehung zu möglichen Depressionssymptomen bei den pflegenden Angehörigen/Freunden gesetzt. Ein zweiter Aspekt betraf mögliche Interaktionswege zwischen diesen Faktoren. Abhängige Variable der Analyse war die vom Patienten wahrgenommene Pflegequalität. Berücksichtigt wurden dabei:

_ die psychosozalen Charakteristika des pflegenden Angehörigen/Freundes

_ die soziodemografischen Charakteristika des pflegenden Angehörigen/ Freundes

_ die Art der Pflege

_ Tumorcharakteristika des Betroffenen — verschiedene Kovariaten und mögliche

Insgesamt ergab sich ein signifikanter Zusammenhang zwischen vom pflegenden Angehörigen angegebenen Depressionssymptomen und der patientenberichteten Pflegequalität: Je höher die De- pressivität, als desto schlechter empfanden die Patienten ihre medizinische Versorgung (Odds Ratio [OR] 1,06, 95\%-Konfidenzintervall [95\%-KI] 0,011,13). Beurteilten die Pflegenden ihre Gesundheit als mäßig oder schlecht, hatten die Patienten eine um mehr als das 3-Fache erhöhte Wahrscheinlichkeit für eine als schlecht wahrgenommene Pflegequalität (OR 3,76, 95\%-KI 1,76-9,55). Wurden psychosoziale Eigenschaften des $\mathrm{Pa}$ tienten, die Qualität der Arzt-PatientenKommunikation und eine koordinierte Patientenbetreuung in der Analyse berücksichtigt, fielen die beobachteten $\mathrm{Zu}$ sammenhänge schwächer aus.

Fazit: Das psychische Wohlbefinden pflegender Angehöriger bzw. Freunde hat deutlichen Einfluss auf die vom $\mathrm{Pa}$ tienten wahrgenommene Qualität seiner medizinischen Versorgung. Die Pflegeperson sollte als ein wichtiges Mitglied im Pflegeteam betrachtet werden und bei gesundheitlichen Problem Unterstützung erhalten. Barbara Kreutzkamp

Litzelman $\mathrm{K}$ et al. How does caregiver wellbeing relate to perceived quality of care in patients with cancer? Exploring associations and pathways. J Clin Oncol. 2016 Aug 29. [Epub ahead of print] 